\title{
An Atherosclerotic Coronary Artery Phantom for Particle Image Velocimetry
}

\author{
Jean Brunette ${ }^{2}$ (jean.brunette $@$ icm-mhi.org) \\ Rosaire Mongrain ${ }^{1,2, *}$ (rosaire.mongrain@mcgill.ca) \\ Adrian Ranga ${ }^{1}$ (adrian.ranga@elf.mcgill.ca) \\ Jean-Claude Tardif ${ }^{2}$ (tardifje@icm.umontreal.ca) \\ * corresponding author \\ ${ }^{1}$ Department of Mechanical Engineering, McGill University, Montreal, Canada \\ ${ }^{2}$ Montreal Heart Institute
}

\begin{abstract}
Myocardial infarction, also known as a heart attack, is the single leading cause of death in North America. It results from the rupture of an atherosclerotic plaque, which occurs in response to both mechanical stress and inflammatory processes. In order to validate computational models of atherosclerotic coronary arteries, a novel technique for molding realistic compliant phantom featuring injectionmolded inclusions and multiple layers has been developed. This transparent phantom allows for particle image velocimetry (PIV) flow analysis and can supply experimental data to validate computational fluid dynamics algorithms and hypothesis.
\end{abstract}

\section{INTRODUCTION}

Myocardial infarction (MI), causing heart attack, is an important cause of mortality and morbidity in the developed countries. Thrombosis (blood coagulation) triggered by the rupture of an atherosclerotic plaque (pathological vessel thickening creating blood flow obstruction) is the most common cause of acute coronary syndromes (myocardial infarction or unstable angina) (17). Understanding the biomechanics of the plaque, along with its physiology, is a key element to understand MI triggering. However, computational studies of blood flow dynamics and its interaction with the vascular wall (fluid-structure interaction: FSI) could provide important information about velocity and pressure fields, and the state of stresses and strains in atherosclerotic coronary arteries. Computational models and their assumptions must be validated in order to verify their accuracy. Realistic hydraulic models (phantoms) of coronary arteries used for PIV are rare. They require being transparent and compliant.

Existing phantoms do not reproduce the architecture of the normal artery layers and their pathological anisotropic inclusions, which strongly affects the global mechanical behavior of the artery and blood flow. Numerical models with simple pathological configuration have been investigated before, although they included neither inclusions, nor the three layers of the artery wall. Ohayon et al (20) have developed a 2dimensional numerical model with multiple layers and inclusions, but when it comes to experimental phantoms of this kind, there is very scarce know-how. Blown-glass tube of uniform thickness has been used for PIV experiment by Grigioni et al (10). Although this model had a realistic shape (it was obtained from magnetic resonance images), it was rigid, monolayer, and they used a water-glycerol fluid, which cannot reach the refractive index of glass. To the best of our knowledge, the only coronary artery phantom featuring a flow disturbance due to pathological flow obstruction (stenosis due to atherosclerosis) with embedded inclusion (pools of pathological tissue like lipid or calcium within the artery wall) has been developed by Kobayashi et al (14) and Tang et al (23). However, this phantom was not transparent (it was developed for echographic-based analysis, therefore not suitable for PIV. Furthermore, it had only one inclusion and did not reproduce the different layers of the artery. Karino et al developed a very elegant technique to render arteries transparent (13). Although they were using a tedious method of manually tracking seeded particles (the ancestor of more recently developed PIV technique), one can imagine that this method of transforming normal tissue into transparent material could be useful for future PIV observation. However the efficiency of this technique applied to pathological tissue remains unexplored and the access to post-mortem arterial segments is neither readily available nor reproducible. Benard et al have been using non-realistic rectangular Plexiglas box to observe, by means of PIV, the flow around a stent-like structure (2). Natarajan et al have been using a transparent cylindrical tube with embedded acrylic bumps for PIV visualization (18). Their model reproduced neither the different layers of the artery wall, nor its pathological flow obstruction (stenosis) and 
therefore flow disturbance. They aimed to reproduce the flow in the vicinity of strut-like bumps. Finally, Bale-Glickman et al have been using for their PIV observation, a silicone phantom molded from an ex vivo atherosclerotic carotid bifurcation (1) geometry (obtained from magnetic resonance images). Their phantom was however mono-layered, of constant thickness, and therefore did not reproduce the wall architecture.

Arteries are composed of three layers, including the intima, the media and the adventitia. The intima is the inner most layer and is very thin. It includes a $5-10 \mu \mathrm{m}$ thick monocellular protection film, called endothelium, which actively prevents the blood from being in direct contact with the artery and its highly thrombogenic content. The intima layer however gets thicker along with the extent of atherosclerotic disease and may develop inclusions that are finite volumes of (typically) lipid (at early stage) or calcium (mature plaques) or any combination. The media is a muscular layer whereas the adventitia is made of connective tissue and microscopic blood vessels called vasavasorum (19).

The molding of the phantom was performed with a set of five molds, where each mold included two halves. All molds were sharing the same male piece, also made of two halves. Each mold reproduced a different layer or inclusion. The aim of this paper is to introduce a novel technique of molding realistic transparent and compliant phantom, featuring injection molded inclusions and all arterial layers for the validation of numerical models and hypotheses.

\section{MATERIALS AND METHODS}

Phantom geometry: It is clear that each plaque has its own original configuration and there is no universal architecture of atherosclerotic coronary artery. The objective of this paper was to introduce a novel method of making transparent realistic phantom for flow visualization more than emphasizing on the specific chosen geometry and dimension. However, based on analysis of a large number of intravascular ultrasound sequences and insights from pathologists' experience, a typical plaque configuration has been modeled.

Lumen narrowing: A 50\% stenosis (flow reduction as compared to sane reference segment), which represents a mild to moderate stenosis, has been chosen since plaques causing infarcts are not the most occlusive. Indeed, plaques demonstrating less than $70 \%$ occlusion are the most vulnerable and infarct related plaques are usually less than $50 \%$ occlusive on the angiography (15). The lumen narrowing was however asymmetric, since significant plaques are usually eccentric (4).

Gaussian shape: This $50 \%$ lumen narrowing was modeled by a bi-Gaussian shape, on the longitudinal and transversal planes. The choice of a bi-Gaussian geometry was a simplification of the complexity of possible lesion configurations. This type of shape is realistic since it can be observed in vivo. Gaussian geometry allows for controlling both width and length of the stenosis by changing parameters into the analytic equation. It also allowed for analytic comparison obtained from computer simulations to that of experimental PIV data. The stenosis, as seen on the transversal plane of the phantom, was semicircular with soft edges that gently came down on the artery wall to avoid abrupt and unrealistic transition between the lesion and the arterial wall, which could have caused geometric singularities. Stress concentration do however exist and can be the result of either geometrical discontinuities (due to the shape of the stenosis) (12) or physiological discontinuities (8) (due to differences of mechanical properties of the different tissues).

Inclusions: Two distinct inclusions were incorporated into the lesion model. The number of inclusions has been arbitrary fixed to a number of two, but the possibility to also study the interaction of two inclusions having different properties was appealing. However, in vivo inclusions have often no clear boundaries, although certain regions have a higher percentage of one type of tissue more than one another. Different types of tissue can commonly be found, like lipids, calcium and fibrosis (also smooth muscle and inflammatory cells). Both inclusions were made of a slightly stained (but transparent) compound for identification purposes.

Dimensions: It was believed that a distance of $3(76 \mathrm{~mm})$ inches (after upscale factor) proximal and distal to the stenosis would allow for a sufficient access and handling of the phantom inside the observation receptacle. An additional straight segment of 16 inches $(406 \mathrm{~mm})$ proximal to the phantom an outside the receptacle insured the flow to fully develop. The reference diameter (without stenosis) was 0.75 inch $(19 \mathrm{~mm})$. A scale-up factor of 6.35 was applied over anatomic dimensions in order to be able to machine and handle the phantom and also to obtain a good resolution on PIV. This factor allowed for a scaled reference diameter of 0.75 inch (19 $\mathrm{mm})$, and inlet and outlet diameters of 1.00 inch $(25 \mathrm{~mm})$, allowing for conventional tubing and adaptor usage. Therefore, the total length of the phantom was 12 inches $(304 \mathrm{~mm})$ including 2 inches $(51 \mathrm{~mm})$ of lesion mimic, 3 inches $(76 \mathrm{~mm})$ of prestenotic segment, 3 inches $(76 \mathrm{~mm})$ of poststenotic segment, and 2 inches $(51 \mathrm{~mm})$ of adaptor segment at both ends to allow perfusion. The adventitia had a thickness of $150 \mu \mathrm{m}$ before the upscale factor, as observed in in vivo intravascular ultrasound (IVUS) images. There is very few information in the literature about the thicknesses of the different layers of the arterial tunica whereas there is much information about its histology. The media had a thickness of $150 \mu \mathrm{m}$ (unscaled). Indeed, media thickness is approximately $200 \mu \mathrm{m}$ or less and the thickness of the adventitia is hard to determine because of surrounding tissues (24). The media thickness however decreases along with the extent of atherosclerosis (9). The intima, which includes the endothelium and the plaque, varied 
from $150 \mu \mathrm{m}$ (unscaled) to $1500 \mu \mathrm{m}$ to reproduce a $50 \%$ stenosis.

Molds machining: The design of the mold was created with a computer assisted design (CAD) software (Pro/Engineer) and all layers and inclusions were assembled in Pro/Engineer. The machining of the molds was performed into aluminum blocs and was done with conventional tools (lathe, milling), whereas certain areas of complex geometry were machined on a computer numerical control (CNC). Numerical data for $\mathrm{CNC}$ was prepared with Master Cam, software that specifies CNC machine mill trajectory.

Phantom molding: First, the silicone T-2 silastic translucent base was prepared by addition of $10 \%$ of $\mathrm{T}-2$ curing agent catalyst (both obtained from Dow Corning Chemicals, MI, USA), which triggered the polymerization of the compound. The mixture was then degassed in a vacuum chamber. Iterative depression-compression cycles were necessary to expand air bubbles and to rupture them respectively. Once most of those bubbles were removed, the compound was spread over the two male and female pieces of the innermost layer set of mold. All parts were then replaced in the vacuum chamber. The second half of the female was placed over the first half, therefore trapping the male in between. The holding and mechanical compression of the whole set of parts for the first molding was secured with a set of 12 allen screws. While assembling the different parts of the mold together with the screws, the previously degassed compound was compressed and the excess material was evacuated by dedicated channels. The vacuum was interrupted during the curing (chemically triggered hardening) in order to avoid any negative-pressure-induced bubble expansion. The mold was then placed in an oven chamber (Fisher Scientific, USA, model Isotemp) previously set at $80^{\circ} \mathrm{C}$ for two hours. Once the silicone was well set, the female parts were removed and the male, along with the set compound, was ready for the next molding step. The second part of the molding was required to create the inclusions and their compounds were also degassed, as previously described, prior to their injection. The male, along with the first intima layer molded on top of it during the first part of the molding (the intima is molded in three steps in order to trap in sandwich the embedded inclusions) was placed into the two female parts of the second mold set. The two compounds were then injected into their respective inclusion channels through the aluminum mold. These channels (small holes through the aluminum mold to inject the pressurized-compound from the syringe to a specific part of the mold) allowed the filling of the respective ellipsoidal cavity and therefore the creation of the inclusions. The silicone mixture was slowly injected (to insure that the air in the mold will be properly evacuated as the mixture fills the cavities) by means of a small diameter syringe $(3 \mathrm{ml}$ luer-lok, Becton Dickinson, NJ) in order to increase the injection pressure (for a given force, if the diameter doubles, the pressure drops by a factor of 4). The compound was injected until the mold cavity was filled and excess material was ejected through the overfill channel. The syringe needle used was of a relatively large diameter (BD 16G1, Becton Dickinson, NJ) to facilitate the flow of the viscous compound once the pressure was built by the syringe. Again, the mold was placed in an oven chamber at $80^{\circ} \mathrm{C}$ for two hours. Once the inclusions were molded, another layer of silicone was added by means of a third set of molds, therefore trapping the inclusions to simulate embedded inclusions into an atherosclerotic plaque. Again, the compound was prepared and degassed, and then spread over the male and both female halves. The mold was closed and placed in the oven chamber for curing purposes. The subsequent molding of the media and adventitia mimics were performed similarly to the previous layers, with their respective compounds and set of molds. Once all layers and inclusions were completed, compressed air was blown through specific channels within the male piece, therefore releasing the phantom from the mold.

Fluid mechanics: Fluid parameters were normalized and adimensionalized, according to Buckingham theorem, in order to properly reproduce physiological perfusion of the phantom. For steady flow, these parameters included consideration of the Reynolds number $(\mathrm{Re})$, that represents the ratio of the inertial over viscous forces $\left(\operatorname{Re}=\frac{\rho u D}{\mu}\right)$, and the Euler number $\left(E u=\frac{P}{\rho u^{2}}\right)$, which is important if pressure is a concern. The Re within a coronary artery $(\sim 200)$ must remain the same within the phantom. This can be achieved by considering the upscaled geometry and by adjusting the amplitude of the flow in conjunction with the viscosity.

Refractive index: The refractive index of silicone was 1.43 and the fluid in which it was perfused and immersed had a similar refractive index in order to avoid optical artifacts during PIV acquisition. A transparent fluid having similar refractive index to that of silicone was created by means of a mixture containing $60 \%$ of glycerol and $40 \%$ of water. The viscosity of that mixture was $14.5 \mathrm{mPa} . \mathrm{s}$ as evaluated with a viscoelastometer (Bohlin Instruments, CVO 120 high resolution, NJ, USA) and the density was $1114 \mathrm{Kg} / \mathrm{m}^{3}$. The Reynolds number of the model was similar to that of coronary arteries $(\mathrm{Re}=194)$, based upon a coronary artery reference diameter of $3 \mathrm{~mm}$ with a $1.53 \times 10^{-6} \mathrm{~m}^{3} / \mathrm{s}$ flow. The phantom was immersed in a transparent enclosure made of polycarbonate.), filled and perfused with the described glycerol mixture, and the bath pressure was adjusted by means of controlled air pressure connected to the observation receptacle. The phantom was also perfused with the same glycerol mixture than the immersion bath, but some particles of titanium $\left(\mathrm{TiO}_{2}\right)$, having a near size of $5 \mu \mathrm{m}$, were included in order to reflect some laser light for displacement tracking purposes. The phantom, enclosure, bath and flow were all transparent, 
therefore allowing appropriate optical conditions for PIV acquisition.). Receptacle walls were perpendicular to the laser beam to avoid optical artifacts like reflection or refraction due to refractive indexes mismatch. Indeed, refraction is governed by the Snell-Descartes law stated as follow: $n_{1} \sin \left(\theta_{1}\right)=n_{2} \sin \left(\theta_{2}\right)$, where $n_{1}$ and $n_{2}$ are the refractive indexes on each side of the interface between two material penetrated by a light beam, and $\theta_{1}, \theta_{2}$ are their respective angle of approach in respect with an axis normal to the interface. Therefore, there is no refraction phenomenon if either the light beam is perpendicular to the interface or the refractive indexes are the same. The compliant phantom was bent in order to study the effect of curvature and torsion on fluid dynamics (secondary flow) and therefore assess the importance for spatially correct 3D reconstruction of coronary arteries (21).

Surrounding tissue: The tissues surrounding the artery do have mechanical consequences on its behavior. The smaller the vessel, the bigger the contribution of the surrounding tissues to the mechanical behavior of the artery (7). The effect of the surrounding tissues was incorporated into the perfused model by addition of an air pressure feature that increased the pressure outside the artery wall. The pressure was adjusted so that the lumen diameter change along the cardiac cycle reached the desired value. This diameter variation decreases with age and degree of atherosclerosis (3), and may vary from $5 \%$, as observed in the physiologic range of atherosclerotic arteries, to $10 \%$ for more compliant vessels (22).

\section{RESULTS}

A set of five complementary molds was created from aluminum blocks. Each mold reproduced either an artery layer or inclusions. Figure 1 illustrates the ten-female pieces and the two male pieces that were machined to mold the phantom. The resulting multi-layer phantom is illustrated in Figure 2. The three layers of the artery were molded along with two inclusions. The molding of the different layers and inclusions was performed with an add-on fashion, where the male-piece of the mold was utilized along the whole molding process. Figure 3 illustrates the throat of the lesion mimic with soft edge geometric transition between the lesion and the wall.

\section{DISCUSSION}

A novel method to create an anatomically correct phantom, compliant and having refractive index that can be matched, featuring injection molded inclusions and reproduction of all arterial layers from a 12-part aluminum mold was developed. Its transparency allowed for laser particle image velocimetry (PIV) and therefore experimental flow visualization for velocity field assessment. Pressure field determination can be calculated from the velocity field with the Navier-Stokes equation.

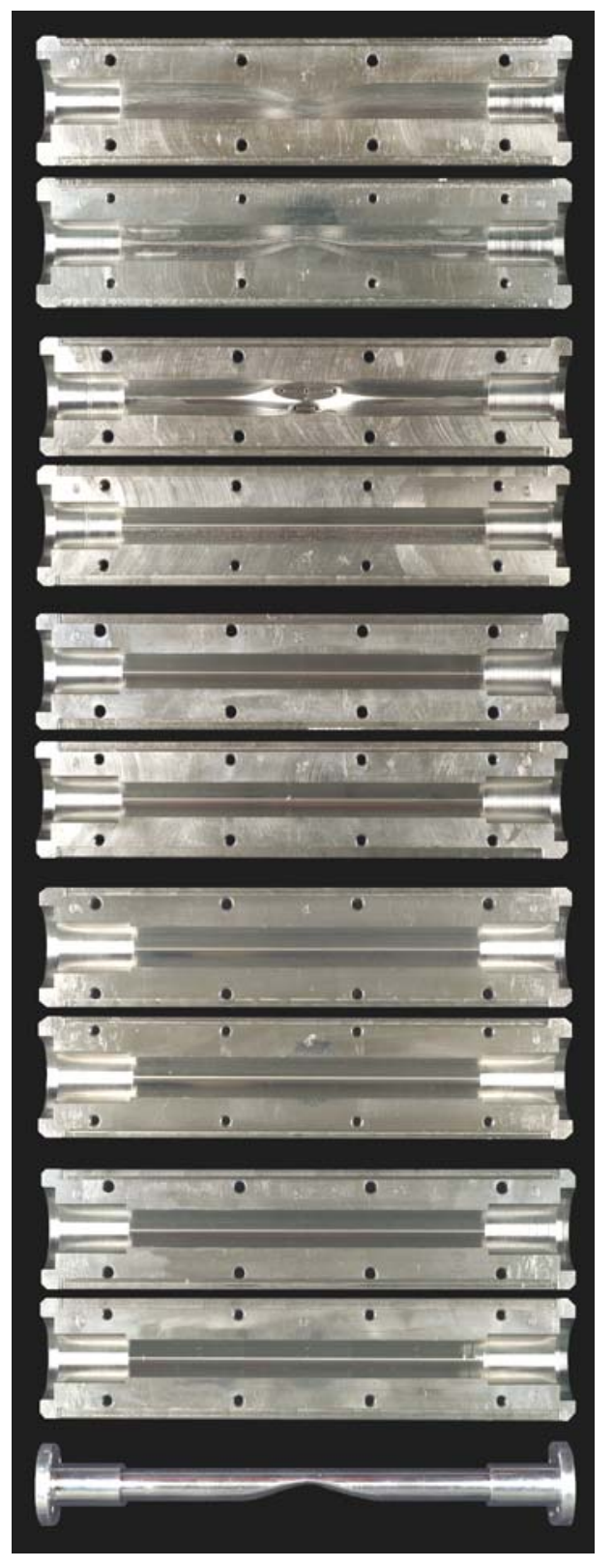

Figure 1: Mold set. Five molds were machined from aluminum blocks, for a total of 10 halves of female molds and a two-part male (lower item). The different layers and inclusions were injection-molded from a silicone-based compound. The male piece was used through the five female molds and the different layers and inclusions were molded in an add-on fashion. The silicone was degassed prior to the molding. The curing was triggered by a catalyst and accelerated by means of heat. 


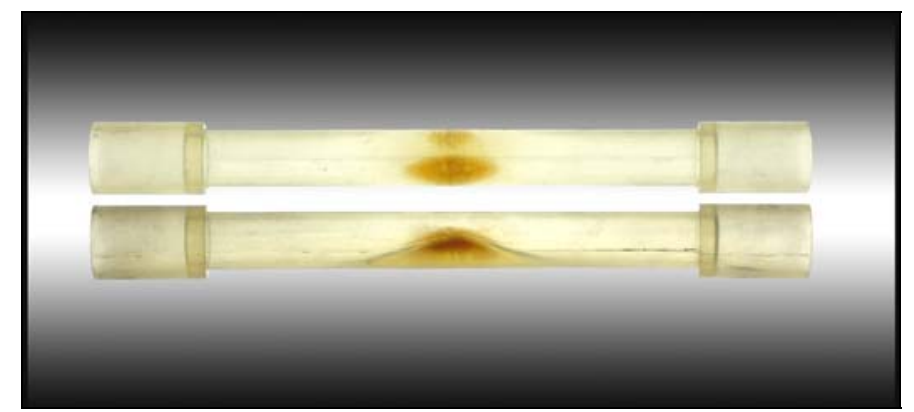

Figure 2: Overall view of the phantom. Longitudinal picture of the molded phantom as seen from top and aside. The two inclusions can be separately identified, as they were slightly stained for visual identification purposes (top view). The outside diameter was constant along the phantom whereas the interior was designed to reproduce a $\mathbf{5 0 \%}$ lumen narrowing. The stenosis had a Gaussian geometry (see bottom view) on the longitudinal plane and was asymmetric on the transversal plane, in order to reproduce a typical plaque. The phantom also featured standard dimensions at its extremities in order to receive a one-inch adaptor for perfusion purposes.

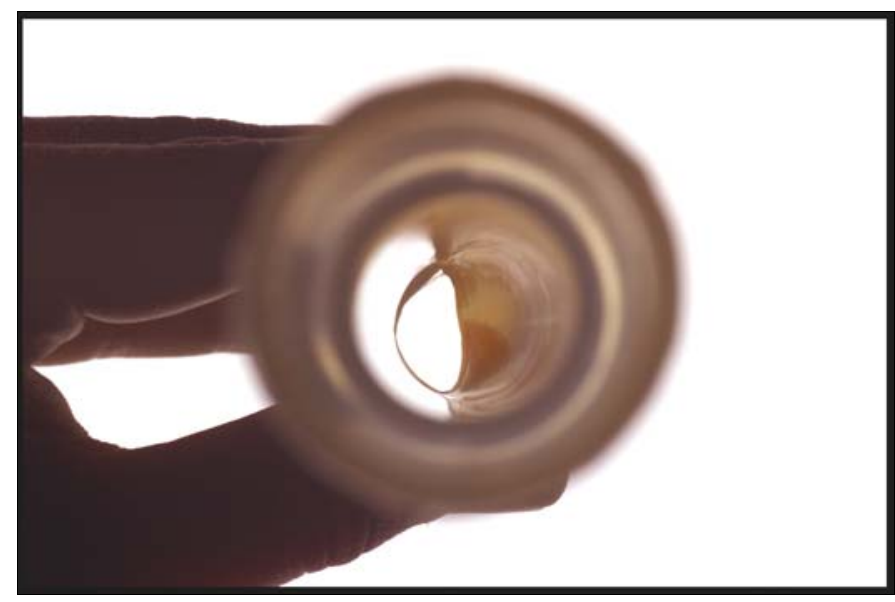

Figure 3. Interior view of the phantom. Cross-section (transversal) of the phantom lumen, revealing the $50 \%$ area stenosis. The junction of the stenosis with the arterial wall was smooth in order to avoid unrealistic stress concentration due to geometric singularities. The slightly stained inclusions can be observed within the stenosis.
Although the method of increasing the outside pressure provided some mechanical effects of the surrounding tissue, it is clear that the imposition of a uniform, isotropic hydrostatic pressure did not perfectly reproduce the complexity of the surrounding tissues. The isotropy of the silicone did not reproduce the oriented arrangement of the artery, especially that of the media, of which muscular fibers are oriented in a tangential fashion, and the complexity of the plaque. The importance of this limitation is however attenuated by the progressive thinning of the media that occurs with atherosclerosis (11). Also, the silicone is a relatively elastic material whereas biological tissues have some viscoelastic behavior. The three-dimensional, time varying material properties and heterogeneous structure of lesions lead to complex biomechanical behavior (16). Our team is presently working on a real size multilayer coronary artery phantom with inclusions, reproducing mechanical properties of in vivo arteries, aimed to test percutaneous transluminal angiography devices. Different materials and additives, like alginate, acrylamide or agar (5), encapsulated or not, are under investigation to improve the different mechanical property possibilities within the phantom. A good reproduction of the plaque mechanical properties will allow to better reproduce the mechanics of plaque rupture (6).

\section{CONCLUSION}

Computational fluid dynamics model hypotheses should be validated with an experimental phantom. A method for creating a transparent coronary artery phantom, featuring injection molded inclusions and multiple layers was developed. The possibility to study blood flow by means of PIV within a transparent atherosclerotic model for different plaques and wall compositions will allow the validation of fluid dynamics software, algorithms and hypotheses. The creation of such visualization phantoms is an important tool for the validation and optimization of cardiovascular device design. 


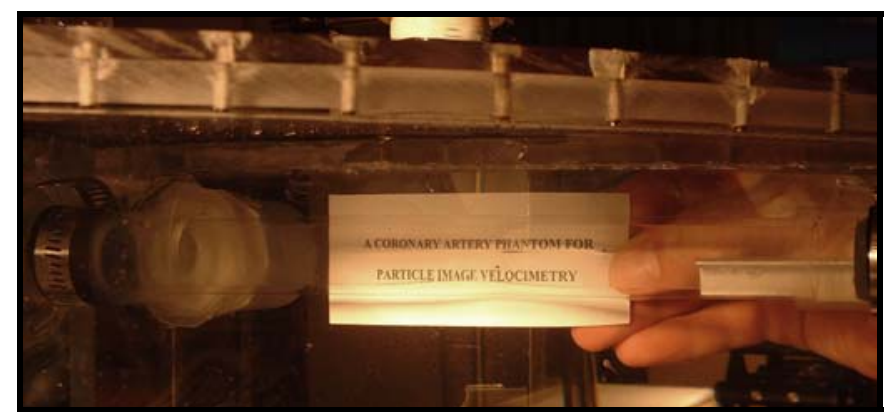

Figure 4: Phantom transparency. All layers and inclusions of the phantom are fully transparent in order to visualize the trajectory of the $5 \mu \mathrm{m}$ seeded titanium oxyde particles. The phantom is submerged into a transparent polycarbonate receptacle containing a mixture of glycerol and water, of which the refractive index matches that of the silicone phantom, in order to avoid any optical artifact like refraction. The phantom is also perfused with the same mixture but containing seeded particles. In this image, a printed peace of paper is held behind the phantom and the receptacle, to demonstrate the transparency.

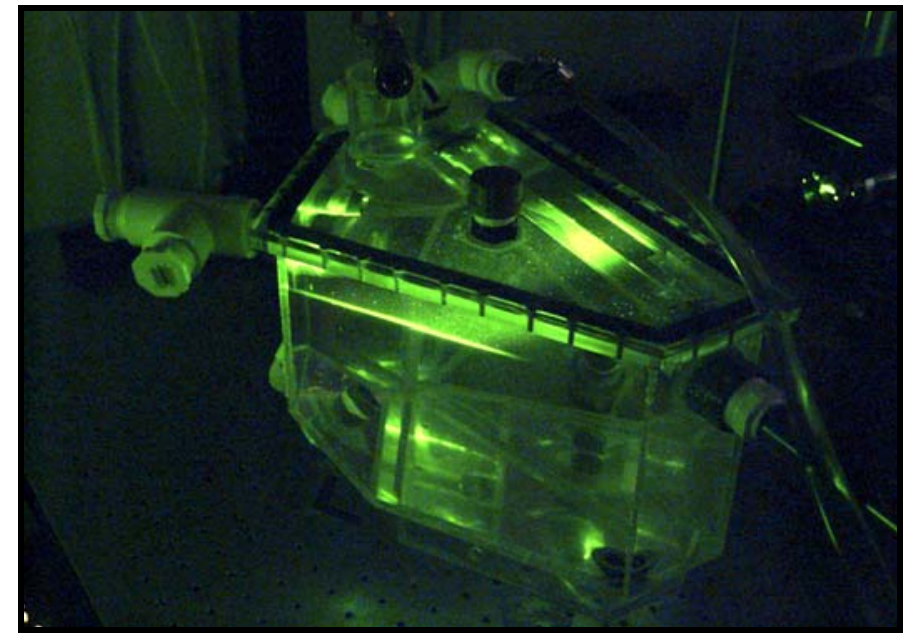

Figure 5: The mounted phantom. Isometric view of the perfused phantom, mounted in its receptacle and bath. The laser beam (coming from the right) illuminated a thin single sheet of the phantom (bright tubular shape) and flow (bright light sheet through the receptacle), allowing the camera to observe the displacement of the $5 \mu \mathrm{m}$ seeded titanium oxyde particles.

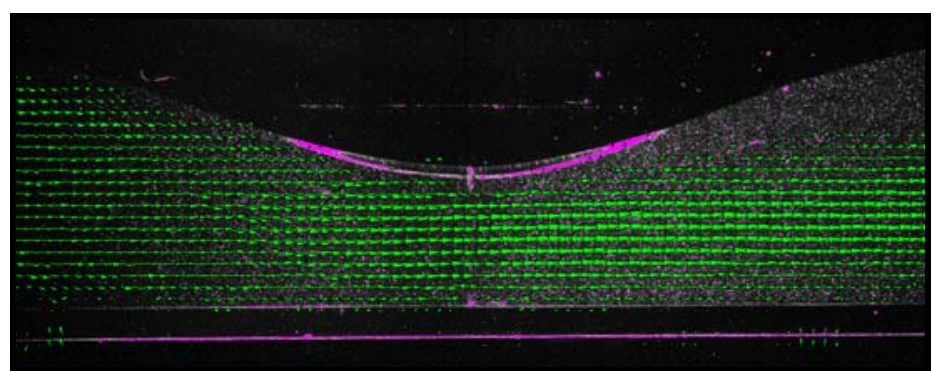

Figure 5: Velocity vectors within the phantom. Particle image velocimetry demonstrated the direction and amplitude of flow within the phantom (small arrows). The laser beam excited a thin layer of the phantom, therefore revealing the trajectory of the seeded tracers within the flow. The stenosis (flow obstruction on the top center) accelerated the fluid downward, proximal (left half) to the throat, whereas deceleration was observed distal (right half) to the stenosis. A zone of slow recirculation was observed on the upper-right section of the flow (no visible arrows because of the low magnitude of the velocity), therefore creating favorable conditions for particle internalization and plaque growth.

\section{REFERENCES}

[1] Bale-Glickman J, Selby K, Saloner D, Savas O. Experimental flow studies in exact-replica phantoms of atherosclerotic carotid bifurcations under steady input conditions. J Biomechanics 2003; 125(1):38-48

[2] Benard N, Coisne D, Donal E, Perrault R. Experimental study of laminar blood flow through an artery treated by a stent implantation: characterisation of intra-stent wall shear stress. J Biomechanics 2003; 36(2003):991-998.

[3] Berry JL, Santamarina A, Moore JE, Roychowdhury S, Routh WD. Experimental and computational flow evaluation of coronary stents. Ann Biomed Eng 2000; 28(4):386-398.

[4] Brown BG, Bolson EL, Dodge HT. Dynamic mechanisms in human coronary stenosis. Circulation 1984; 70(6):917-922.

[5] Brunette J, Mongrain R, Cloutier G, Bertrand M, Bertrand OF, Tardif JC. A novel realistic three-layer phantom for intravascular ultrasound imaging. Int J Card Imaging 2001; 17:371-381.

[6] Brunette J, Mongrain R, L'Allier PL, Bertrand OF, Grégoire J, Tardif JC. Intravascular imaging and the biomechanics of plaque rupture. Journal of clinical engineering 2003; 28(3):163-173.

[7] Comolet R. Biomécanique circulatoire. Masson ed 2-225-80278 5.Paris: 1984

[8] Felton CV, Crook D, Davies MJ, Oliver MF. Relation of plaque lipid composition and morphology to the stability of human aortic plaques. Arterioscler Thromb Vasc Biol 1997; 17:1337-1345.

[9] Greenleaf JF. Tissue characterization with ultrasound. CRC Press ed 1986.

[10] Grigioni M, Amodeo A, Daniele C, D'Avenio G, Formigari R, Di Donato RM. Particle image velocimetry analysis of the flow field in 
total cavopulmonary connection. Artificial organs 2000; 24(12):946

952.

[11] Gussenhoven EJ, Frietman P, The SH, van Suylen RJ, van Egmond F, Lancée CT, Van Urk H, Roelandt J, Stijnen T, Bom N. Assessment of medial thinning in atherosclerosis by intravascular ultrasound. Am J Cardiol 1991; 68:1625-1632.

[12] Gyongyosi M, Yang P, Hassan A, Weidinger F, Domanovits H, Laggner A, Glogar D. Coronary risk factors influence plaque morphology in patients with unstable angina. Coronary Artery Dis 1999; 10:211-219.

[13] Karino T, Motomiya M. Flow visualization in isolated transparent natural blood vessels. Biorheology 1983; 20:119-127.

[14] Kobayashi S, Tsunoda D, Fukuzawa Y, Morikawa H, Tang D, Ku DN. Flow and compression in arterial models of stenosis with lipid core. ASME 2003;(2003 summer bioengineering conference, june 25 29, Florida):497-498.

[15] Kullo IJ, Edwards WD, Schwartz RS. Vulnerable plaque: pathobiology and clinical implications. Ann Intern Med 1998; 129:1050-1060.

[16] Lee RT. Atherosclerotic lesion mechanics versus biology. Z Kardiol 2000; 89(Suppl.2):II-80-II-84.

[17] Lee RT, Grodzinsky AJ, Frank EH, Kamm RD, Schoen JF. Structure-dependent dynamic mechanical behavior of fibrous caps from human atherosclerotic plaques. Circulation 1991; 83(5):1764 1770 .

[18] Natarajan S, Mokhtarzadeh-Dehghan MR. A numerical and experimental study of periodic flow in a model of a corrugated vessel with application to stented arteries. Med Eng Phys 2000; 22(2000):555-566.

[19] Netter FH. The CIBA collection of medical

illustrations. Yonkman F, editor. 0-914168-07-X 1987.

[20] Ohayon J, Teppaz P, Finet G, Rioufol G. In-vivo prediction of human coronary plaque rupture location using intravascular ultrasound and finite element method. Coronary Artery Dis 2001; 12:655-663.

[21] Reiber JCH, Dijkstra J, Koning G, Oemrawsingh PV, Schalij MJ, Goedhart B. Current and future developments in QCA and image fusion with IVUS. What's new in cardiology imaging? Kluwer academic publisher. 1998: 17-21.

[22] Shaaban AM, Duerinck AJ. Wall shear stress and early atherosclerosis: a review. Am J Radiology 2000; 174:1657-1665. [23] Tang D, Yang C, Kobayashi S, Ku DN. Steady flow and wall compression in stenotic arteries: A three-dimensional thick-wall model with fluid-wall interactions. J Biomed Eng 1 A.D.; 123:548 557.

[24] Tardif JC, Lee HS. What's new in cardiovascular imaging? Applications of intravascular ultrasound (IVUS) in cardiology. Kluwer academic publisher 0-7923-5121-5, 1998: 133-148. 